\title{
THE TREATMENT OF PENETRATING STAB WOUNDS OF THE CHEST
}

by J. M. Hayse-Gregson, Dip. Phys. (U.C.T.) Physiotherapist, Baragwanath Hospital.

Those of us who have had to treat patients with penetrating stab wounds of the chest, will appreciate that physiotherapy plays a vital role in the management of these patients. At Baragwanath Hospital Proximately one thousand five hundred Non-European utients with stabbed chests are admitted yearly. In addition these patients might present with a stabbed sbdomen requiring a laparotomy, while not infirequently, patients are admitted with a penetrating stab into the heart, necessitating a thoracotomy. However, for the purpose of this article the treatment of patients with penetrating stab wounds of the chest requiring intercostal drainage only, is discussed.

\section{Mechanism of Injury}

The intrapleural pressure within the chest is less than stmospheric pressure, varying from approximately minus nine to minus twelve centimetres of water in inspiration, and from minus three to minus six centimetres of water on expiration. Thus expansion of the lungs is passively maintained by a partial vacuum within the pleura. When $\downarrow$ stab wound is inflicted which penetrates the pleural cavity, allowing air to enter, the negative pressure within the pleural space is eliminated, resulting in collapse of the lung, the degree of collapse being proportional to the amount of air in the pleural space. Pressure within the pleural space may increase progressively until it exceeds atmospheric pressure, contituting a severe tension-pneumothorax. This may arise when the laceration to the chest wall, trachea, bronchus or smaller bronchiole presents as a 'flap valve' which allows the free passage of air into the pleural space on inspiration, but traps the air within the pleura on expiration. This increasing pressure within the pleural space will not only totally collapse the lung on the affected side, but will cause the mediastinum to shift to the opposite side, which in turn will produce compression of the good lung. Mediastinal shift and deviation of the trachea can be clearly seen on X-Ray. This state of affairs will not reverse until an intercostal drain is inserted, which will allow the air within the pleural space to escape. The rate at which the lung expands is not proportional to the extent of the pneumothorax, but depends on how quickly the damaged lung tissue seals itself. Pleural thickening and the presence of other pathology in the lung will retard expansion. Clinical signs of pneumothorax are:

(i) Deareased air entry.

(ii) Hyper-resonance on percussion.

(iii) Deviation of the trachea away from the affected side. (See Fig. 1.)

When the lung tissue or the intercostal vessels are lacerated, the patient presents with a haemothorax. Laceration of lung tissue is the most common cause of a haemothorax, when the clotting of vessels is usually rapid and effective, thus rendering secondary haemorrhage or a progressively increasing haemothorax an unusual occurrence. Nevertheless a second X-Ray will fre- 


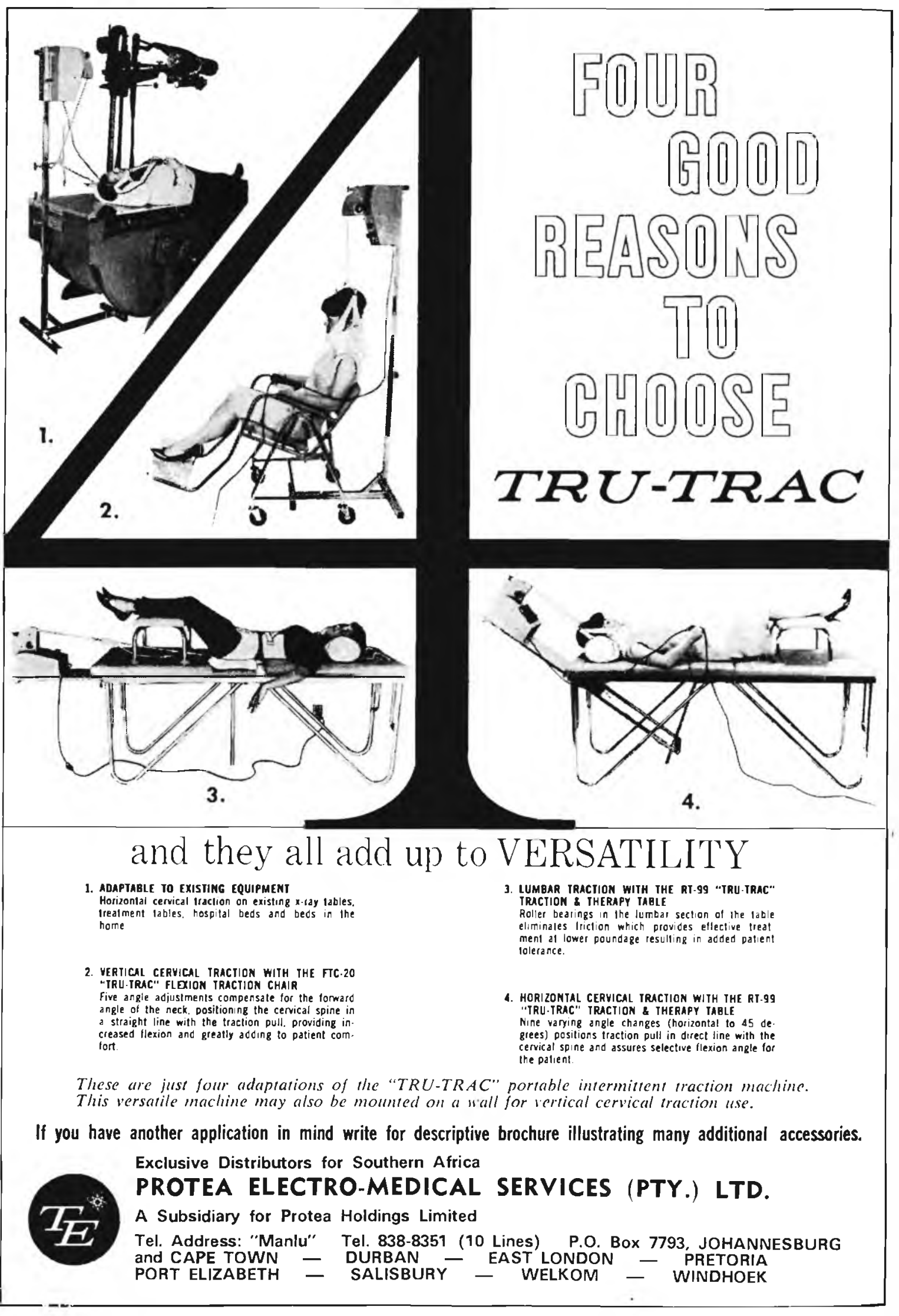




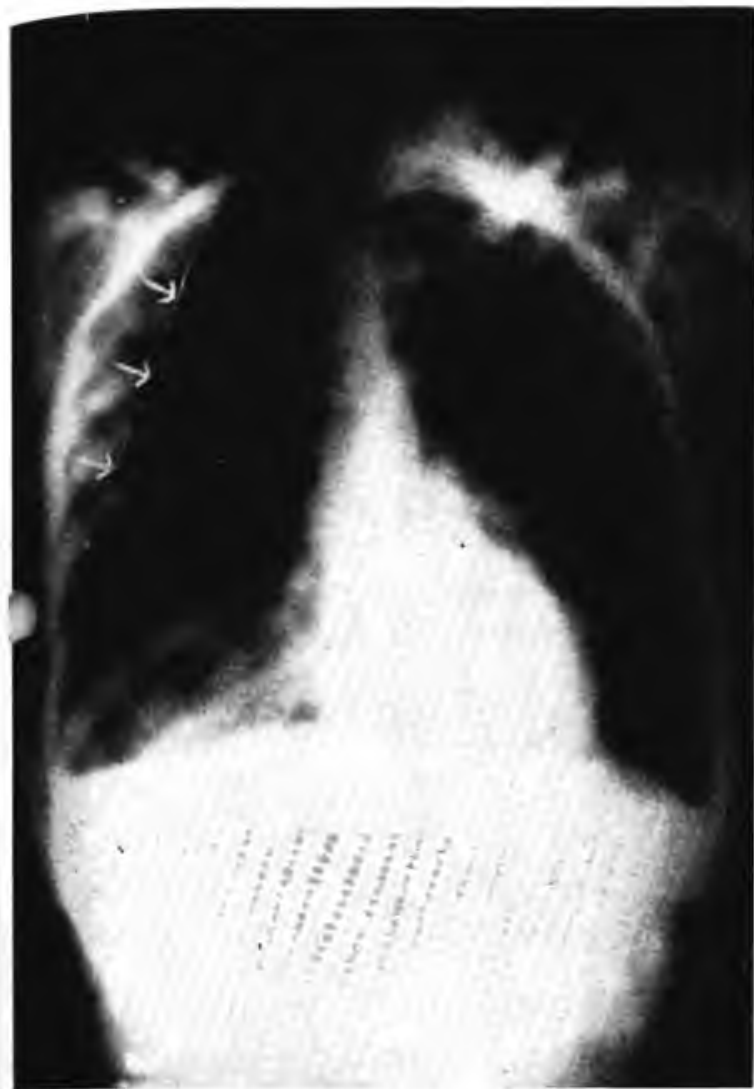

Fig 1. X-Ray showing right sided pheumothorax. Arrows indicate outline of partially collapsed lung.

quently reveal an increased amount of fluid in the chest. This is usually due to blood in the pleural space acting as an irritant thereby calusing the pleura to produce a serous exudate. The clinical signs of a haemothorax are:

(i) Dullness on peicussion.

(i)

Decreased ait cntry.

(iii) Dyspnoea

(iv) Shock.

(v) Pain.

Patients most commonly present with a haemopneumothorax, although the one condition may be present without the other. (See Fig. 2.)

At Baragwanath Hospital, the extent of a haemothorax or pneumothorax is expressed as a percentage of blood or air in the affected side of the chest. This is not an accurate assessment but serves as a rough guide as to the severity of haemo or pneumothorax. and is classified according to figures 3 and 4 . (See Figs. 3 and 4.)

It should be noted that a $15 \%$ to $20 \%$ haemothorax. which will appear on X-Ray as an obliteration of the costo-phrenic angle will represent approximately one and a half pints of fluid within the adult chest. It is often surprising how much fluid can be drained from a chest, which on X-Ray appeared to contain only a mall amount. is it takes about five hundred cc's of blood in the pleural cavity to produce, radiological and physical evidence of a haemothorax. A haemothorax of less than $15 \%$ is usually treated conservatively - i.e. without intercostal drainage.
Clinical Picture and Patho-Physiology:

The clinical picture may include pain, respiratory distress and shock. and to a greater or lesser extent shows the following signs:

(i) Pallor.

((i) Cyanosis.

(iii) Tachypnoea

(iv) Dyspnoea

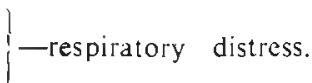

(v) Hypovolemic shock - i.e. raised pulse rate, fall in blood pressure.

Pallor and clinical shock are usually due to loss of blood. but are often greater than would be expected for the amount of blood actually lost. The fall in blood pressure stimulates the cardiac centre. which brings about an increase in heart rate. Respiratory distress is produced by a number of factors. The elimination or decrease of the negative pressure normally existing in the pleural cavity diminishes venous return, and this. plus the reduced volume of circulating blood. results in reduced cardiac output. De-oxygenated blood pumped to the lungs is only effectively oxygenated by one lung. as the affected lung is partially or totally collapsed. In addition, injury to the lung itself, to the chest wall, and possibly to the diaphragm, result in pain and lack of effective movement of the affeoted side of the chest.

\section{Intercostal Drainage:}

Intercostal drains work on the simple principle of a water seal, as per diagram. (See Fig. 5.)

This allows the free passage of blood and air down the tube from the pleural cavity. Provided the botitom of a tube (a) in the diagram is under the water level

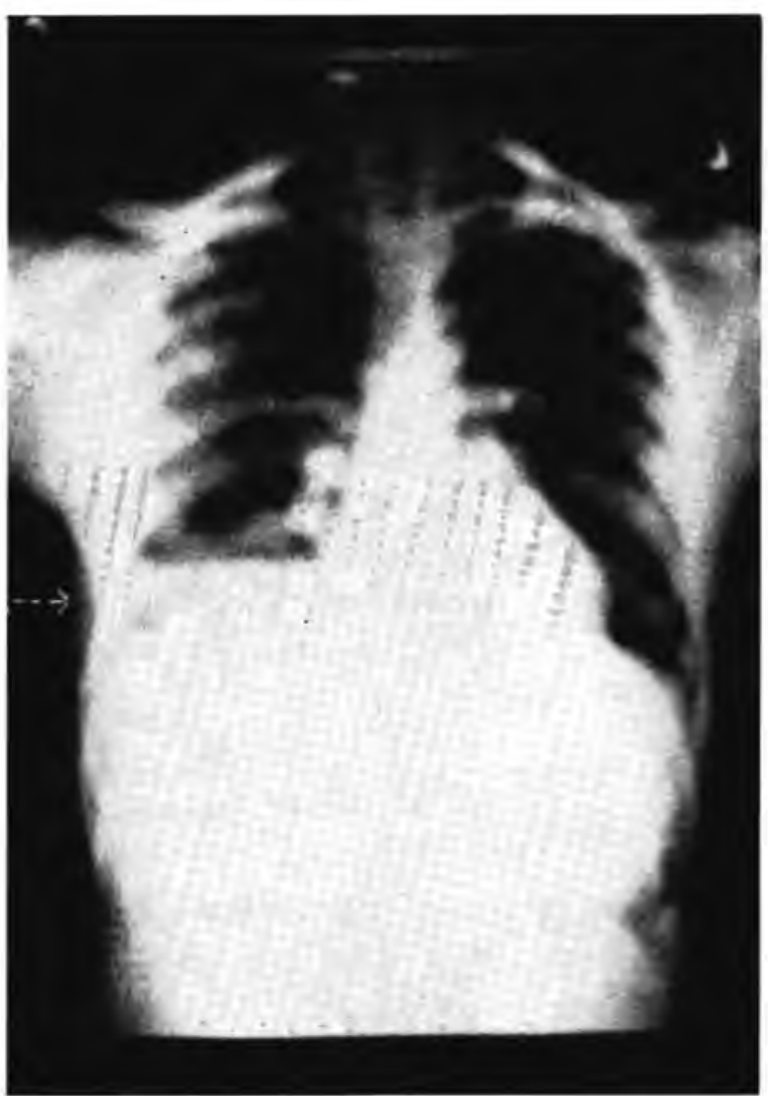

Fig. 2. X-Ray showing right sided haemothorax, indicated by broken arrow. 


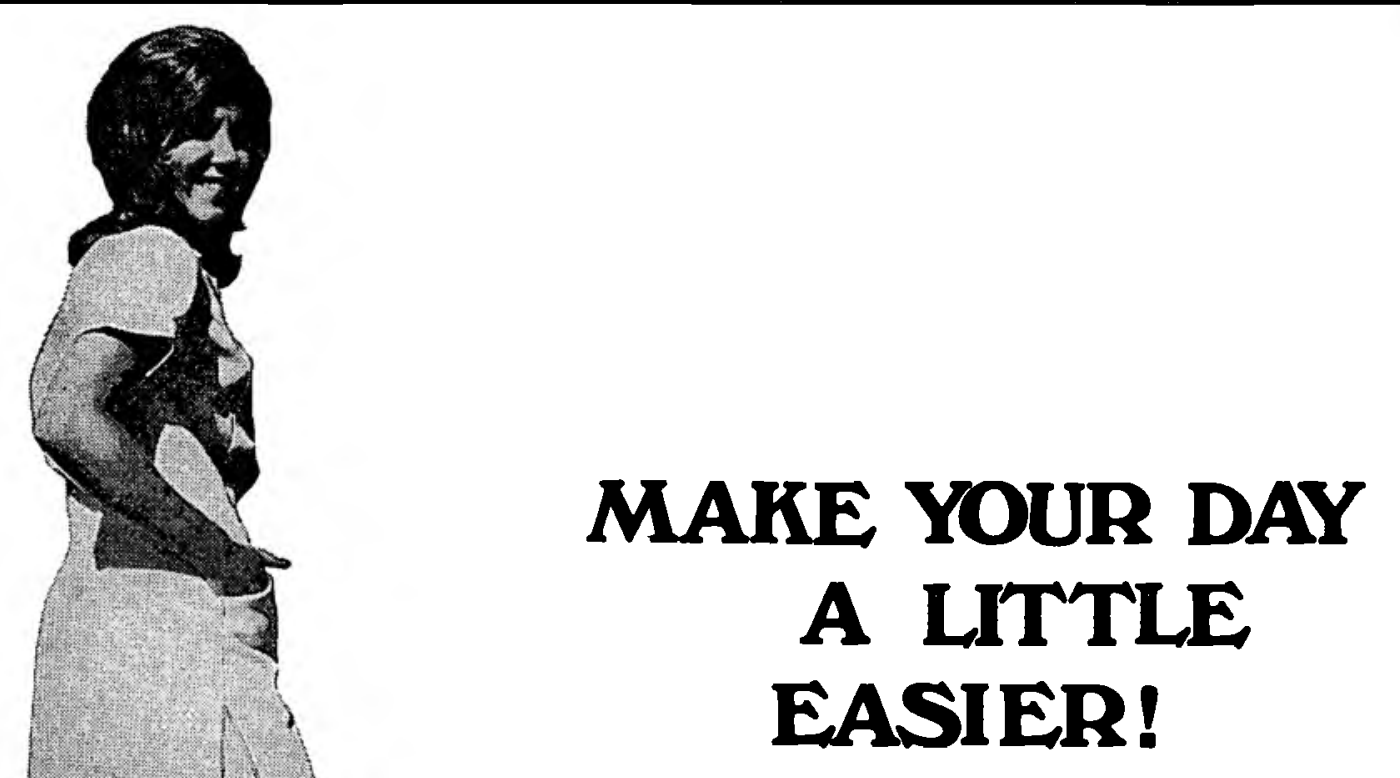

You physios are a hard-worked group, we know. In the day's work, environment and comfort become more and more important - and while there's not much we can do to help fix the background in which you work, you'll find a comfy, practical working rig in the new specialpurpose uniform we've designed with you in mind.

It's a continental style dress, with Bermuda-type shorts to match. A side vent in the skirt and the generous cut, mean that you'll work more easily, and feel comfortable right through the hard day.

Your option of short or long sleeves, in drip-dry, hard-wearing fabric... white or saxe blue. Sizes 32 to 42 .

Elegance and efficiency ... what more could you ask?

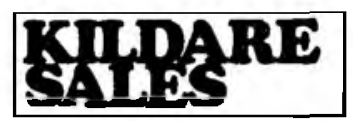

4th Floor, Pritchard House, 83 Pritchard Street, JOHANNESBURG. Telephone 23-4405.

87 Marion Avenue, Glenashley, DURBAN. Telephone 83-7226.

Fully illustrated catalogues and price lists of our full range are available free to you. Just drop us a postcard (P.O. Box 752, Johannesburg) or telephone 23-4405 any time, including nights, weekends and holidays. We're also happy to execute phone orders, of course. 


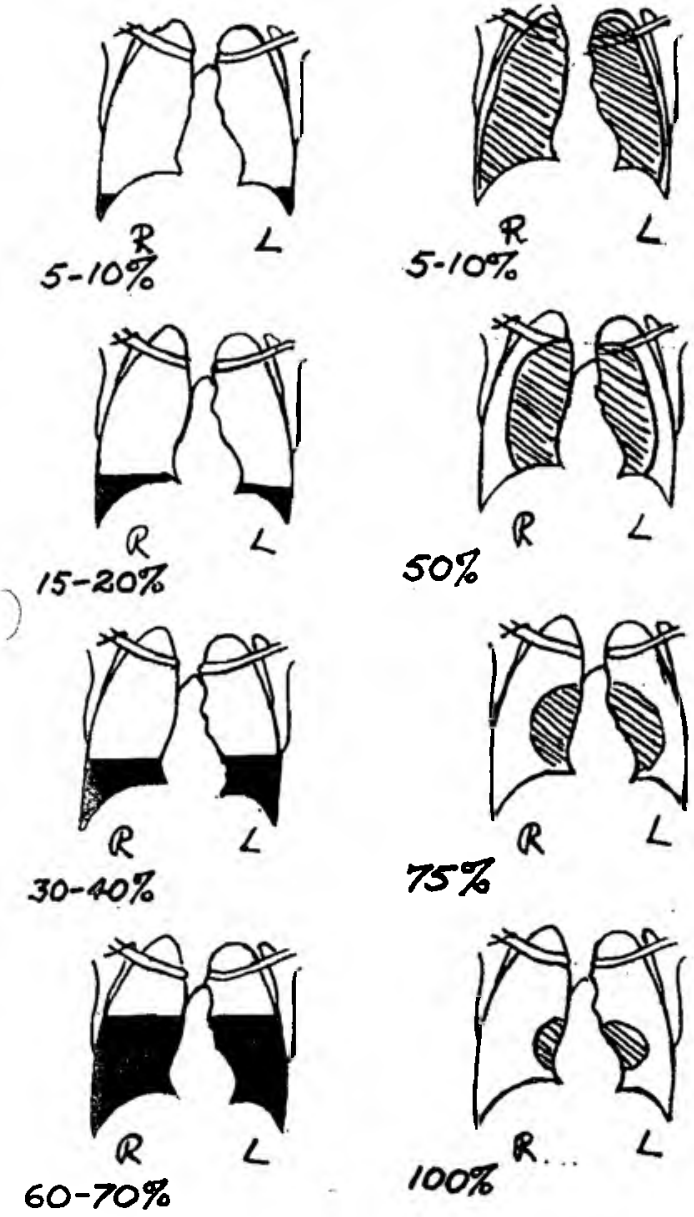

Fig. 3. Assessment of pneumothorax.

Fig. 4. Assessment of haemothorax.

in the bottle, air cannot travel up the tube. As air is removed from the patient's chest, and the negative issure therein is re-established, the water level in tube a) will rise slightly. This level swings when the patient breathes or coughs, and will continue to do so until the lung is re-expanded. Provided the tube is patent, the degree of fluctuation of the water level within the tube is a sensitive and accurate indicator as to the extent of re-expansion. The amount of blood drained can be accurately measured. (Figs. 6 and 7 .)

Patients presenting with a broncho-pleural or alveolarpleural fistula are placed on negative suction, attached to tube (b) in Fig. 5. This condition manifests by excessive bubbling in the drain bottle on breathing and coughing, and failure of lung expansion. Generally, suction is applied from one to three days and is removed when there is no further bubbling, and expansion is maintained.

\section{Treatment:}

Prior to World War II, the practice was to aspirate just enough fluid to relieve the dyspnoea, and to replace the fluid removed with air. The reasoning behind this Was that compression of the injured lung would prevent further bleeding. The patients were on strict bed rest, and the chest was strapped to avoid excessive movement. Coughing and unnecessary activity were strictly avoided.
Nowadays the principles of treatment are somewhat reversed, the emphasis being on early evacuation of the haemothorax, and re-expansion of the lung as soon as possible.

\section{On Admission:}

The patient is first treated for existing shock by immediately replacing lost blood by means of intravenous fluid. The patient is then routinely $X$-Rayed. Depending on the extent of the haemopneumothorax, an intercostal drain is inserted under local anaesthesia, and the patient is then re-X-Rayed. Physiotherapy is commenced as soon as possible after insertion of the drain.

\section{Aims of Physiotherapy:}

1. To obtain early expansion of the lung.

2. To bring about effective drainage of the haemothorax as soon as possible, before the blood clots within the chest.

3. To prevent accumulation of secretions in the lungs, thereby preventing atelectasis, pneumonia, etc.

4. To prevent complications assooiated with the presence of an intercostal drain (particularly with long term drains, such as with an empyema) i.e. postural deformity, wasting of the arm and shoulder girdle muscles of the affected side due to disuse, and splinting of the affected side of the chest.

\section{Methods:}

1. Breathing exercises-(a) diaphragmatic breathing.

(b) localised breathing to the affected side.

bilateral lateral costal breathing.

2. Coughing.

3. Active exercise - arm and shoulder exercises, thoracic mobility exercises, vigorous activities, e.g. running. jumping, etc.

4. Postural drainage and percussion if necessary.

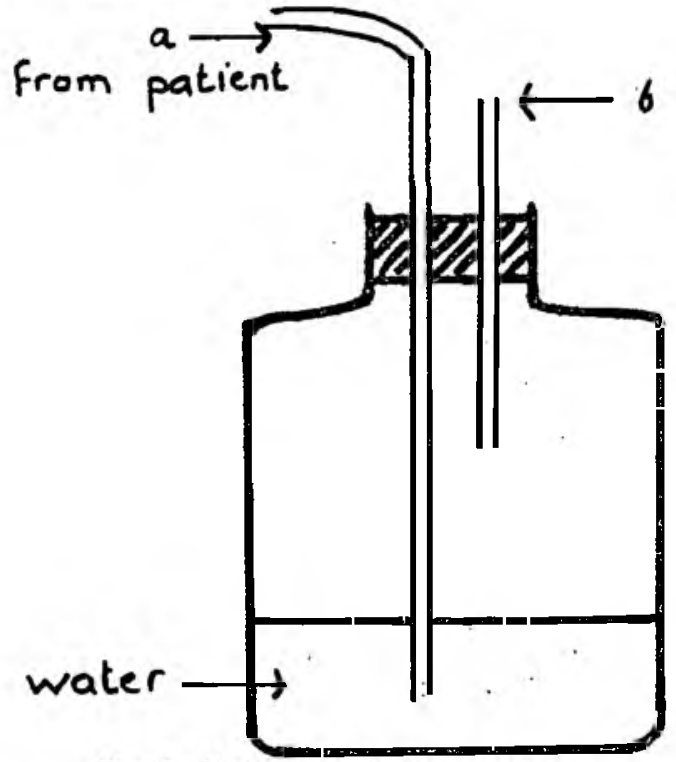

Fig. 5. Underwater drainage system.

The majority of patients seen at Baragwanath Hospital are young and otherwise fit individuals, whose chests prior to injury are normally clear. However, because the lung responds to collapse by increasing secretions, many patients will have a productive cough following the injury. Postural drainage is not given as a routine, and only to those patients with excessive secretions, or persistent atelectasis. Coughing consti- 


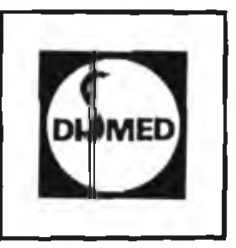

THE

PRESENTS - Minidyne mk III

TRANSISTORISED FARADIC UNIT

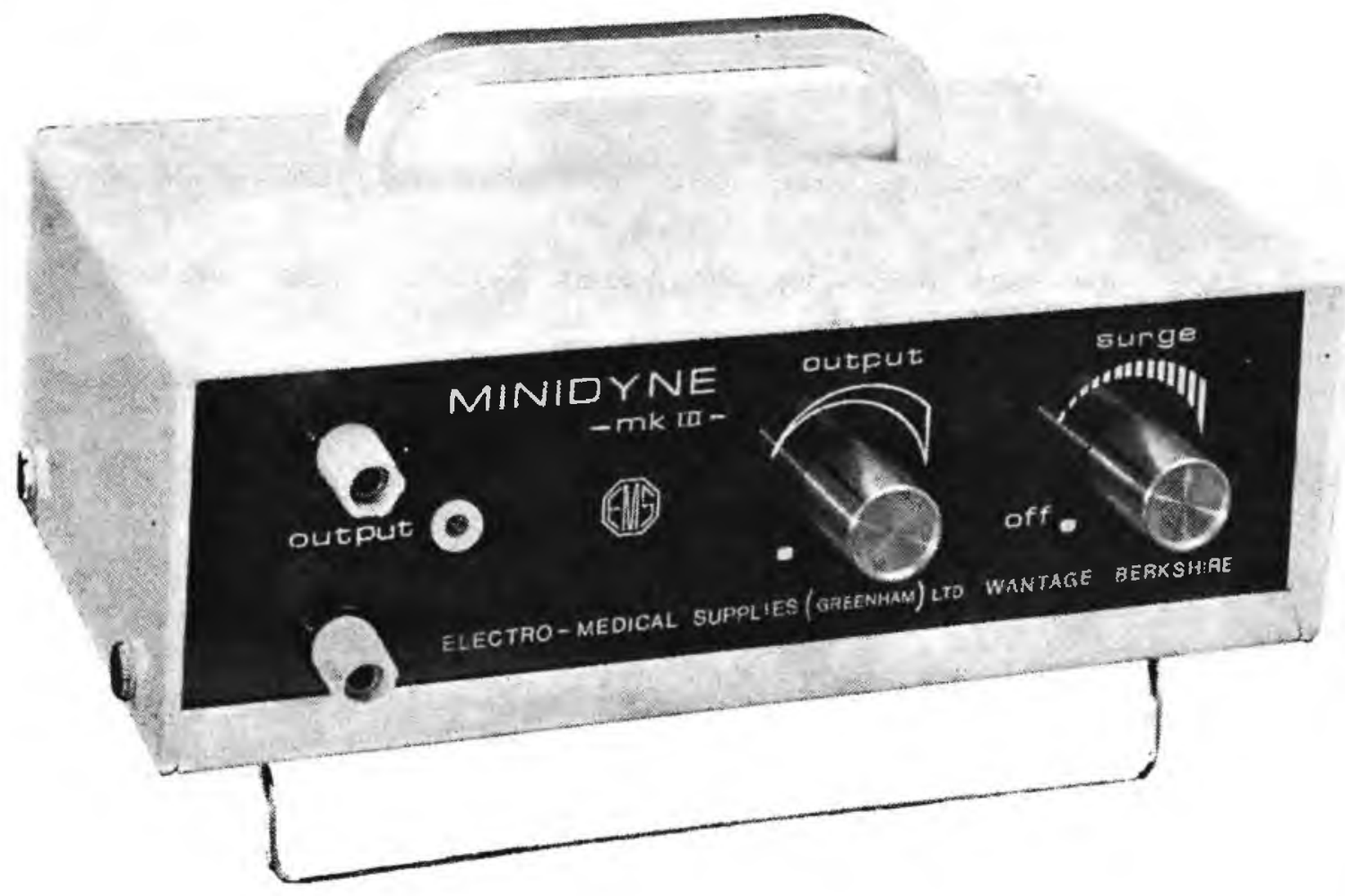

Representing another advance in the evolution of the Minidyne, the Mark III version incorporates printed circuits which reduce the size but also enhance the reliabilsty; continuing features are

wide range of surge speed control with output sufficient for all forms of faradic techniques

audible as well as visual indication of surge speed enabling the operator to anticipate a muscle reaction without having to watch the control panel

fitted with Ever Ready PP9 batteries which will give up to six months' use without replacement and are obtainable worldwide

PRICE:

$\mathbf{R} 87,50$

WEIGHT reduced to: $4 \mathrm{lbs}$. $(1,8 \mathrm{~kg})$

SIZE reduced to $8^{\prime \prime} \times 5 \frac{1}{2} " \times 2 \frac{1}{2}{ }^{\prime \prime}(20 \mathrm{~cm} \times 13,5 \mathrm{~cm} \times 6 \mathrm{~cm})$. complete with accessories.

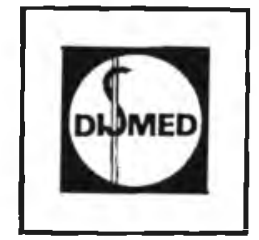

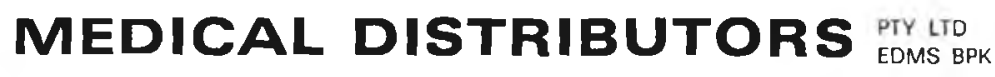

\begin{tabular}{l|l|l|l}
\hline CAPE YORK' | 252 JEPPE ST. | JOHANNESBURG | De Waal House. 172 Victoria Road | Woodstock. C.P. \\
\hline PLEASE ADDRESS ALL CORRESPONDENCE TO P.O. BOX & 3378 JOHANNESBURG \\
RIG ASSEBLIEF ALLE KORRESPONDENSIE AAN POSBUS & \\
\hline TEL. ADD. 'DISMED' & PHONE | FOON 23-8106 & TELEX: $43-7129$ S A. \\
\hline
\end{tabular}


the suction is removed, and the patient joins the chest class.

Occasionally, in spite of intensive physiotherapy, there is the occasional patient whose lung fails to reexpand completely, or who still presents with markedly decreased air entry on the affected side. We have found that giving these patients breathing exercises (diaphragmatic and localised breathing to the affected area) on the Bennett respirator generally achieves good results. A mixture of air and oxygen is administered at a pressure of between fifteen and twenty cms. of water. (Figs. 6 and 7.)

\section{Complications:}

The most common complications following a penetrating stab wound of the chest are atelectasis, and a clotted haemothorax. The latter should be suspected when the amount of blood drained or aspirated from the chest is less than would be expected on X-Ray evidence. While the occurrence of a clotted haemothorax may be due to inadequate drainage of ineffectual physiotherapy, it is not necessarily so. A small percentage of cases develop a clotted haemothorax in spite of vigorous and effective physiotherapy. Clotting of blood within the chest is a phenomenon not fully understood. It may occur within an hour or two of injury, or after a few days. At Baragwanath Hospital a series of experiments was performed on patients with a clotted haemothorax, taking venous blood, and establishing the clotting time. It was found that the clotting time was no shorter than normal, and bore no relation to the speed with which the haemothorax clotted.

A late clotted haemothorax consists of the following:

1. An outer fibrinous layer adherent to the pleura.

2. A layer of varying thickness of unorganised fibrin.

3. A central localised cavity containing fluid.

The outer fibrinous layer becomes organised, and is invaded by capillaries, and fibroblasts. The extent of these changes depends on the age of the clot, and is well advanced within seven days. Progressive invasion of fibroblasts into this layer results in an increasingly thickened fibrinous layer, which will become mature fibrous tissue after approximately four weeks. This thick, tough inelastic fibrous tissue prevents re-expansion of the lung. Eventually the pleura itself becomes thickened. The presence of an empyema accelerates these changes.

Such changes necessitate surgical intervention. In the first two to three weeks a pleural toilet is performed when the clotted material and the fibrinous layer are removed. If the fibrinous layer is not removed within two to three weeks, it becomes fixed to the pleura necessitating a decortication.

\section{Survey of Cases:}

A survey of stabbed chest cases was carried out at Baragwanath Hospital over a four month period during the latter part of 1971. Cases were selected at random firom three of five similar surgical units. Only those cases presenting with an uncomplicated haemothorax and/or pneumothorax requiring intercostal drainage were selected, i.e. patients with additional serious injuries such as penetrating stabs of the abdomen or heart were excluded from this survey. The aim of the survey was purely to analyse the results of routine treatment carried out at Baragwanath Hospital.

Of the one hundred and fifty cases $92,7 \%$ were male, only eleven $(7,3 \%)$ being female. The average age of patients was twenty-four and a half years, the youngest being a boy of fourteen years, and the oldest a man of sixty-five years.

Sociological analysis showed that $67,6 \%$ of cases were unmarried. The occupations of the patients observed ranged from clerks, unemployed, to prisoners.
The majority were manual labourers. It was found that observations relying on information from the patients themselves were unreliable. For instance, of a hundred and fifty cases, only fifty-two $(34,6 \%)$ admitted to being drunk on admission. The percentage of patients admitted in this condition is in fact considerably higher.

The most common weapon used was a knife (in one hundred and forty-one cases). Eight patients were stabbed with screwdrivers, and one was assaulted with a small chopper. $77,7 \%$ of cases presented with a haemopheumothorax. Four cases $(2,7 \%)$ had bilateral pneumothoraces. The average duration of intercostal drainage was 2,1 days, and the average stay in hospital was 3,6 days. The average number of physiotherapy treatments given during the stay in hospital was 5,3 .

\section{Incidence of Complications:}

of one hundred and fifty cases, $2,7 \%$ required reinsertion of the intercostal drain, i.e. four patients. Two of these required aspirations or multiple aspirations in addition to re-insertion of the drain. In all, seven cases $(4,7 \%)$ required aspiration after the initial intercost2? drain was removed. Three of these patients required more than one aspiration.

Included in the above were two patients who refused physiotherapy. One was discharged after three days, but re-admitted a day later when an intercostal drain was re-inserted. Following removal of the drain, one hundred and fifty cc's of blood were aspirated. The patient, still extremely un-co-operative, was discharged six days later, with fluid still present on X-Ray.

Only one patient developed pneumonia. He was admitted to hospital two days after being assaulted. The pneumonia resolved within a few days, on active physiotherapy and antibiotics. One other patient developed pleuirisy.

Broncho-pleural or alveolar-pleural fistulae occurred in ten cases. All these were treated on negative suction as described. Of this group, the average stay in hospital was 4,8 days, as against 3,6 days in patients with no fistula.

Only one patient required a thoracotomy. The indication for this was persistant bleeding four days after insertion of the drain, and persistantly marked decreased air entry of the affected lung. A pleural toilet was performed, and the patient was discharged after ten days, with good air entry on the affected side. Two cases had a known history of pulmonary T.B.- It was interesting to note that their rale of progress was not noticeably affected by the presence of this pathology, being in hospital five and four days respectively, and their drain remaining in situ four and two and a half days respectively. Fifteen of the cases had had previous admissions to hospital for penetrating stab wounds of the chest. Of these, six had had intercostal drains on the same side as the present injury. Surprisingly, this fact did not seem to affect their rate of recovery, as none of these patients had the drain in situ for more than three days. nor were any of the six hospitalised longer than five days.

\section{Condition on Discharge:}

\section{4 normal on $X$-Ray}

32 had a small residual haemothorax

3 had a small pneumothorax

1 had a small haemopneumothorax

Thirty of a hundred and fifty patients had decreased air entry of the affected lung at the time of dischairge and three of these had some underlying consolidation

\section{Conclusion:}

It has been our experience that the rate of progress of these patients is governed very largely by two faotors: (i) By the efficacy of the physiotherapist concerned.

(ii) By the degree of co-operation on the part of the patient. 
The latter point is well shown by the unsatisfactory post-traumatic course of one of the two patients who refused physiotherapy. It is essential that these patients are encouraged to perform their exercises effectively. which often necessitates a somewhat aggressive approach.

The survey showed that the time interval between admission and the commencement of physiotherapy to be rather long. It would be interesting to compare the above results with those achieved in patients treated immediately after insertion of the drain. Hence it is reasonable to assume that a physiotherapist on night duty, who would see the patients immediately after insertion of intercostal drainage would prove very beneficial. On the other hand, due to the fact that a considerable number of cases are admitted in a drunken state, co-operation from such cases would be minimal.

Finally, the effective treatment of chest wounds requires that the physiotherapist be able to assess X-Ray changes, and be able to use a stethescope. The latter permits instant recognition of effective expansion and Carance of seoretions, and also allows the physioderapist to detect areas of consolidation and atelectasis, enabling her to vary her treatment more intelligently.

\section{REFERENCES:}

EDWARDS, F. R. Foundations of Thoracic Surgery (Edinburgh: Livingstone, 1966.)

$H O O D, R . M$. (Ed.). Management of Thoracic Iniuries. (Springfield: Thomas, 1969.)

JOHNSON, J. Surgery of the Chest, 3rd edition. (Chicago: Year Book Medical Publishers, 1964.)

SHEFT, L. M. Initial Management of Thoracic and Thoraco-Abdominal Trauma, 2 nd edition by Hewlett. (Springfield: Thomas, 1968.)

$B L A D E S, B$. Surgical Diseases of the Chest, 2nd edition. (St. Louis: Mosby, 1966.)

A. STEIN, F.C.S., and G. Schnier, D.M.R.D. 'Penetrating Stab Wounds of the Chest' - South African Medical Journal, 3rd July, 1965, No. 24, Vol. 39.

\section{ACKNOWLEDGMENTS:}

I would like to thank the Superintendent of Baragwanath Hospital for permission to publish this article. My thanks are also due to Professor $R$. Lipschitz and to $M r$. B. Rabinowitz for their helpful advice, to $M r$. Melvyn Penn for printing the photographs, and to members of the Physiotherapy Department of Baragwanath Hospital for their co-operation. 